\title{
Statistics of Turbulence in the Stable Boundary Layer Affected by Gravity Waves
}

\author{
Zbigniew Sorbjan • Agnieszka Czerwinska
}

Received: 28 November 2012 / Accepted: 9 February 2013 / Published online: 3 March 2013

(C) The Author(s) 2013. This article is published with open access at Springerlink.com

\begin{abstract}
In order to investigate effects of interactions between turbulence and gravity waves in the stable boundary layer on similarity theory relationships, we re-examined a dataset, collected during three April nights in 1978 and in 1980 on the 300-m tower of the Boulder Atmospheric Observatory (BAO). The BAO site, located in Erie, Colorado, USA, $30 \mathrm{~km}$ east of the foothills of the Rocky Mountains, has been known for the frequent detection of wave activities. The considered profiles of turbulent fluxes and variances were normalized by two local, gradient-based scaling systems, and subsequently compared with similarity functions of the Richardson number, obtained based on data with no influence of gravity currents and topographical factors. The first scaling system was based on local values of the vertical velocity variance $\sigma_{\mathrm{w}}$ and the Brunt-Väisäla frequency $N$, while the second one was based on the temperature variance $\sigma_{\theta}$ and $N$. Analysis showed some departures from the similarity functions (obtained for data with virtually no influence of mesoscale motions); nonetheless the overall dependency of dimensionless moments on the Richardson number was maintained.
\end{abstract}

Keywords Boulder Atmospheric Observatory · Gradient-based similarity · Gravity waves $\cdot$ Stable boundary layer

\section{Introduction}

Turbulence in the stably stratified boundary layer often coexists with gravity (buoyancy) waves (e.g. Turner 1983; Einaudi and Finnigan 1993; Cuxart et al. 2000; Yagüe et al. 2001). Gravity waves can be related to shear (Kelvin-Helmholtz) instabilities (e.g. Blumen et al.

\section{Z. Sorbjan}

Department of Physics, Marquette University, Milwaukee, WI 53201-1881, USA

e-mail: zbigniew.sorbjan@mu.edu

Z. Sorbjan · A. Czerwinska ( ()

Institute of Geophysics, Polish Academy of Sciences, Warsaw, Poland

e-mail: aczerwinska@igf.edu.pl 
2001), density currents (e.g. Sun et al. 2002), and triggered by small-scale topographic features (e.g. Nappo 1991). When waves are sufficiently steep, they can overturn and generate their own turbulence and mixing. Wave effects are usually detected on temporal and spatial records of in situ and remote sensors as fluctuations or undulations of pressure, temperature, reflectivity, and refractive index, with periods in the range from about a minute to about an hour, and wavelengths from $100 \mathrm{~m}$ to several km (e.g. Rees et al. 2000).

Typically, the wave motions are not clearly distinguishable from the turbulent fluctuations (e.g. Hunt et al. 1985). Unfiltered gravity waves, however, can cause various statistics of turbulence to be significantly different than analogous moments associated with genuine, small-scale turbulence. Including mesoscale effects in calculated fluxes can degrade similarity relationships (Smedman 1988). Mahrt et al. (2012) described a method for differentiating between turbulent fluctuations and gravity waves, based on a variable-averaging width. Within the method, the perturbations were divided into three categories: "turbulent" with $0-5 \mathrm{~s}$ time scales, "somewhat turbulent" with 5-60 s scales, and those with little "characteristics of turbulence", i.e. with time scales >60 s. Caughey and Readings (1975), Lu et al. (1983), and Hunt et al. (1985) discussed the effects of waves in terms of spectral analysis.

The primary intention of this study is to extend the analysis of Hunt et al. (1985), hereafter referred to as HKG-85, and to compare the observed moments of turbulence, which include the effects of gravity waves, with the estimates based on the gradient-based similarity functions of the Richardson number. The similarity functions were derived by Sorbjan (2010), and by Sorbjan and Grachev (2010), using data collected over a flat Arctic ice cap during the Surface Heat Budget of the Arctic Ocean (SHEBA) experiment, with no influence of gravity currents and topographical factors.

Our paper has the following structure. The profiles of the potential temperature, wind hodographs, as well as profiles of fluxes and variances, are presented in Sect. 2. The comparison of the similarity functions with the observed statistics of turbulence is discussed in Sect. 3, and final remarks are presented in Sect. 4.

\section{Observations}

\subsection{Data}

The analysis of HKG-85 was based on observations collected in nocturnal conditions on the 300-m tower of the Boulder Atmospheric Observatory (BAO). The site, located in rolling terrain in Colorado, USA, $30 \mathrm{~km}$ east of the foothills of the Rocky Mountains and about $60 \mathrm{~km}$ from the Continental Divide, has been known for its frequent detection of wave activities, triggered by density currents and topographic features. The vicinity of the BAO is characterized by a local terrain slope of about $7 \%$, while the foothills have roughly a meridional orientation.

Data analyzed by HKG-85 were summarized in tables appended to their paper, and contained values of the wind velocity and direction, temperature, dew-point temperature, the vertical velocity and temperature variances, as well as the momentum and temperature fluxes, detected at eight levels of the BAO tower: 10, 22, 50, 100, 150, 200, 250, and $300 \mathrm{~m}$. The tower instrumentation at each level included three-axis anemometers, propeller-vane anemometers, fast-response platinum wire and slow-response quartz thermometers, and cooled-mirror dewpoint hygrometers. The sampling rate for the turbulence sensors was $10 \mathrm{~s}^{-1}$, and $1 \mathrm{~s}^{-1}$ for the mean profile sensors. The standard averaging interval for the mean wind and temperature statistics was 20 or $60 \mathrm{~min}$. 
Table 1 Data summary of the cases analyzed by Hunt et al. (1985)

\begin{tabular}{|c|c|c|c|c|c|}
\hline Event & Date & Period (MST) & $d d\left(^{\circ}\right)$ & $L_{*}(\mathrm{~m})$ & Waves \\
\hline 1 & 18 April 1978 & $2120-2140$ & 053 & 148 & Moderate \\
\hline 2 & 18 April 1978 & $2220-2240$ & 049 & 132 & Present \\
\hline 3 & 18 April 1978 & $2300-2320$ & 342 & 33 & Present \\
\hline 4 & 22 April 1978 & 0006-0026 & 253 & 702 & Present \\
\hline 5 & 22 April 1978 & 0026-0046 & 265 & 212 & Present \\
\hline 6 & 22 April 1978 & 0046-0106 & 276 & 16 & Present \\
\hline 7 & 22 April 1978 & $0206-0226$ & 286 & 850 & Strong \\
\hline 8 & 22 April 1978 & 0226-0246 & 276 & 189 & Strong \\
\hline 9 & 22 April 1978 & 0246-0306 & 280 & 133 & Strong \\
\hline 10 & 15 April 1980 & $1700-1720$ & 276 & 123 & Weak \\
\hline 11 & 15 April 1980 & $1820-1840$ & 303 & 36 & Present \\
\hline 12 & 15 April 1980 & 1900-1920 & 258 & 18 & Present \\
\hline
\end{tabular}

The considered observations were collected on three nights: on 18 and 22 April 1978, and on 15 April 1980. HKG-85 selected twelve 20-min events, which we will refer to as E1-E12. Their characteristics are summarized in Table 1, which lists the event names, dates, hours of occurrence, wind direction $d d$ and the Monin-Obukhov (MO) length $L_{*}=u_{*}^{2} /\left(\kappa \beta T_{*}\right)$, evaluated at $z=10 \mathrm{~m}$, where $u_{*}$ is the friction velocity, $T_{*}$ is the surfacelayer temperature scale, $\kappa$ is the von Karman constant, and $\beta=g / T$ is the buoyancy parameter.

Events 1-3 took place on the evening of 18 April 1978, in three intervals, 2120-2140, 2220-2240, and 2300-2320 MST, with Event 1 classified by HKG-85 as the case with "moderate waves". Waves during Events 2 and 3 were not categorized by HKG-85, and wave activities for these cases are named in Table 1 as "present". Events 4-6 were observed on the night of 22 April 1978, in the intervals 0006-0026, 0026-0046, and 0046-0106 MST. The wave activity was present, but not classified by HKG-85. Events 7-9 took place on the same night of 22 April 1978, in the intervals 0206-0226, 0226-0246, and 0246-0306 MST. The events were assigned as cases with "strong waves".

Events 10-12, on the night of 15 April 1978, included data collected at 1700-1720, 18201840, and 1900-1920 MST, with Event 10 classified as the case with "weak waves". Events 11-12 were not categorized by HKG-85, and wave activities for these cases are marked in the Table 1 as "present".

As it follows from Table 1, the wind direction $d d$ at the level of $10 \mathrm{~m}$ was in the range 253-342 ${ }^{\circ}$ during Events 3-12, and 049-053 ${ }^{\circ}$ during Events 1 and 2. The Obukhov length $L_{*}$ at $z=10 \mathrm{~m}$, was between 16 and $850 \mathrm{~m}$, with the largest values of $L_{*}$ observed during Events 4 and 7.

HKG-85 focused their analysis on spectra, cospectra, quadrature spectra, correlations, and length scales. The momentum and temperature fluxes, as well as variances of the vertical velocity and temperature, were evaluated, but not analyzed by them. These authors found that in the "weak-wave" case, there was no apparent wave energy in the $w$ spectrum, but the $\theta$ spectrum showed a weak concentration of energy in bands of frequency. The $w \theta$ cospectrum and quadrature spectrum were not much different from those in purely turbulent motions. With "moderate-wave" activity, concentrations of energy appeared at discrete frequencies in the spectra. The cospectrum was of the same order as the quadrature spectrum, with peaks roughly at the same frequencies. The "strong-wave" events showed significantly more energy at low frequencies in the $w$ spectrum, and inertial subrange levels were comparable with those 
during the day. The spread between $10-\mathrm{m}$ and $150-\mathrm{m}$ spectral peaks was more pronounced than in the moderate wave case. The $\theta$ spectrum showed distinct maxima, which corresponded roughly with peaks in the $w$ spectrum. Peaks in the $w \theta$ cospectrum and quadrature spectrum were closer together on the frequency scale. Near the low end, the cospectrum changed sign, which could have been associated with internal waves (as the heat flux due to internal waves at times may be counter-gradient).

In all events, a significant amount of energy of internal wave motion was mixed with turbulence, while heat was transferred vertically by low-frequency turbulence, and also by wave or wave-like motions.

\subsection{Evaluation of $\Theta$ and $R i$}

Since the potential temperature was not provided in HKG-85, we estimated it from the definition, $\Theta=T(1000 / p)^{0.286}$. The pressure $p$ was evaluated by employing the hydrostatic balance, which leads after integration to the exponential dependence of pressure on height in the form: $p(z)=p_{0} \exp \left[-g z /\left(R T_{0}\right)\right]$, where $p_{0}=840 \mathrm{hPa}$ is the mean pressure at Boulder, $g=9.81 \mathrm{~m} \mathrm{~s}^{-2}$ is the gravity acceleration, $R=287 \mathrm{~m}^{2} \mathrm{~s}^{-2}, \mathrm{~K}^{-1}$ is the gas constant, $T_{0}$ is the ground-level temperature, and $z$ is the altitude. The effects of humidity were neglected.

In order to evaluate gradients, profiles of the wind velocity components $U$ and $V$, as well as the potential temperature $\Theta$, were approximated using analytical expressions of the form: $f(z)=A+B z+C z^{2}+D \ln (z)$. The coefficients $A, B, C$, and $D$ were found by using the method of the least squares, and the resulting expressions were subsequently used to evaluate the Richardson number $R i=N^{2} / S^{2}$, where $N=(\beta \mathrm{d} \Theta / \mathrm{d} z)^{1 / 2}$ is the BruntVäisäla frequency, $\beta$ is the buoyancy parameter, $S=\left[(\mathrm{d} U / \mathrm{d} z)^{2}+(\mathrm{d} V / \mathrm{d} z)^{2}\right]^{1 / 2}$ is the wind shear.

To improve the overall accuracy of approximations, some single points (outliers) had to be omitted during the fitting process, as e.g., for the potential temperature during Event 11 at the level of $100 \mathrm{~m}$, and during Event E12 at the level of $300 \mathrm{~m}$. Similarly, the wind components above the level of $150 \mathrm{~m}$ during Events 1 and 2 were not taken into consideration. When the approximated values and the observational points significantly differed, the second-order finite differences were used to evaluate the temperature and wind-velocity gradients. Specifically, the finite differences were employed to evaluate gradients of the wind components (not shown) during Event 3 (at the level of $50 \mathrm{~m}$ ), Event 10 (at the levels of 150 and $200 \mathrm{~m}$ ), Event 11 (at the levels above $150 \mathrm{~m}$ ), and for the Event 12 (at the level of $100 \mathrm{~m}$ ).

\subsection{Potential Temperature and Wind Profiles}

The observational points and the approximating curves of the potential temperature $\Theta$ are presented in Fig. 1. Note that the wind direction at the level of $10 \mathrm{~m}$ during Events 1-2 was $049-053^{\circ}$, which implies easterly winds off the plains toward the mountains (Table 1). During Events 3-12, the wind direction was in the range $253-342^{\circ}$, which indicates westerly winds off the mountains. During Event 3 the wind direction near the surface changed to $342^{\circ}$, and a $3 \mathrm{~K}$ surface cooling within 20 min occurred (Fig. 1a), which can be associated with cold-air advection. The surface cooling during Events 4-9 (Fig. 1b) was significantly lower, about $2 / 3 \mathrm{~K} \mathrm{~h}^{-1}$. A relatively strong, over $3 \mathrm{~K}$ surface cooling within $40 \mathrm{~min}$ during Events 10-12 (Fig. 1c) can be associated with cold-air advection due to the drainage flow. 

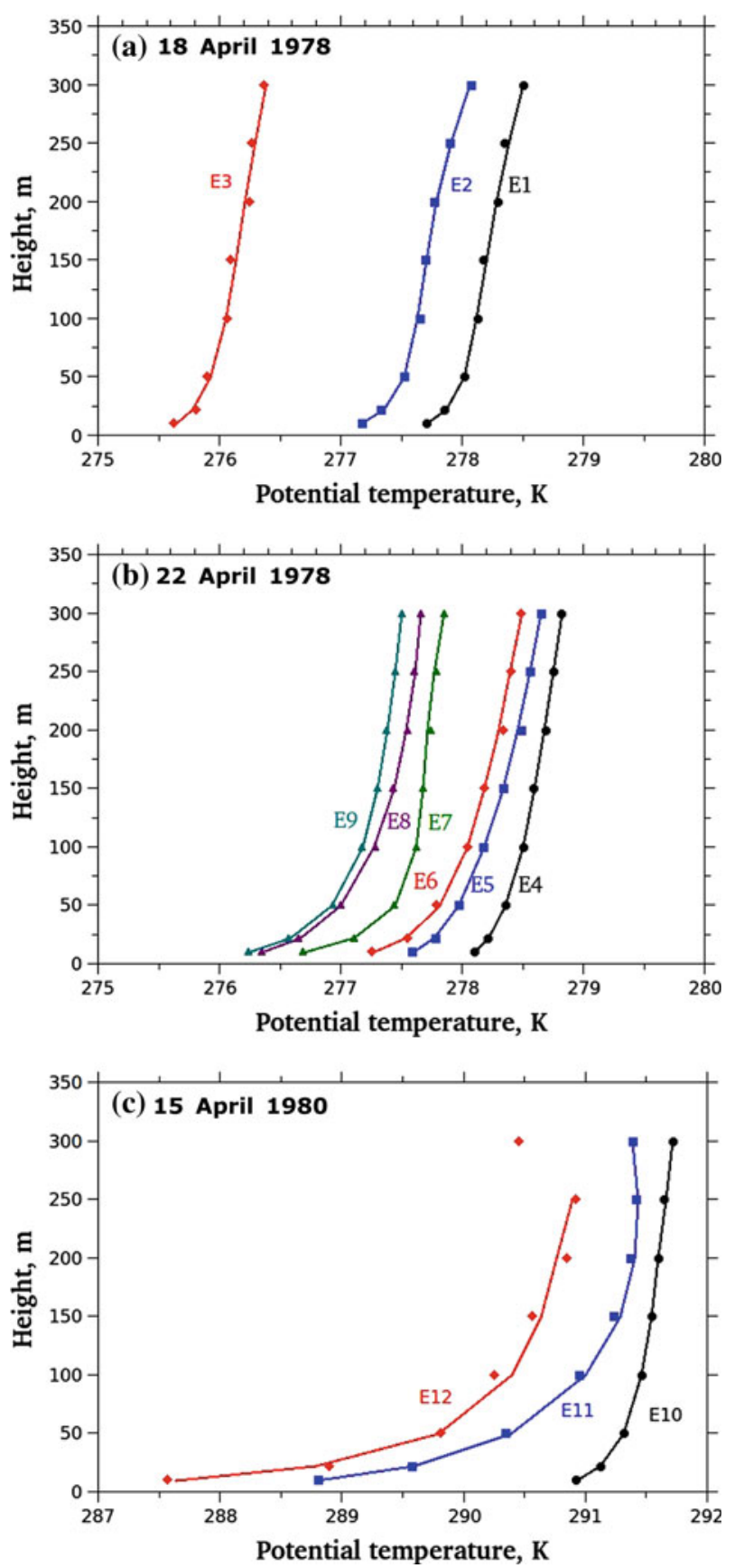

Fig. 1 Observational points and approximating curves of the potential temperature $\Theta$ during: a Events E1-E3, b Events E4-E9, c Events E10-E12. The highest data point in case E12 was disregarded 


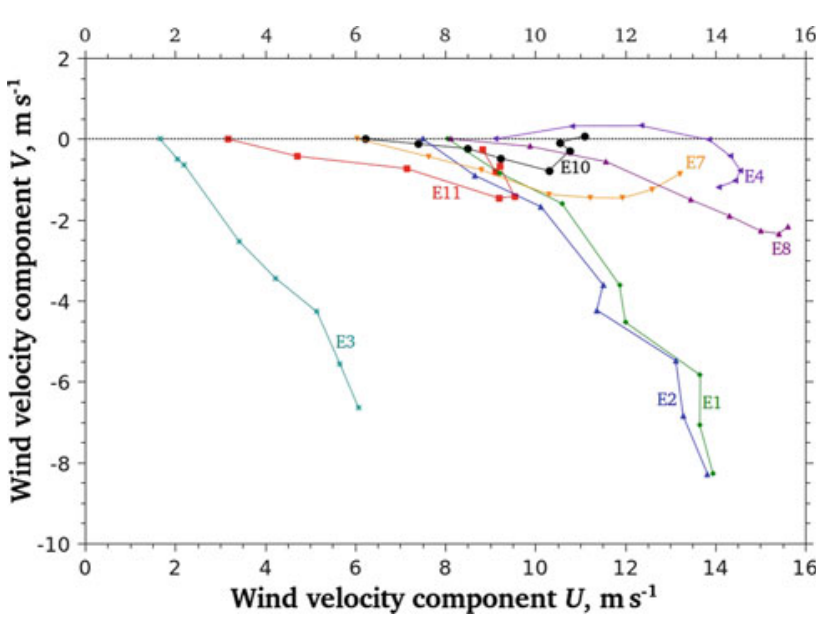

Fig. 2 Wind hodographs during Events E1-E4, E7, E8, E10 and E11. The $x$-axis of the coordinate system is oriented along the wind direction at the lowest level of $10 \mathrm{~m}$. The highest level is $300 \mathrm{~m}$

Profiles of the wind velocity are shown in HKG-85, and consequently are not displayed here. Instead, we present selected wind hodographs (for E1-E4, E7, E8, E10, and E11) in Fig. 2. The $x$-axis of the coordinate system is oriented along the wind directions at the lowest level of $10 \mathrm{~m}$. The hodographs for Events 1-3 are spiral, with the angle $\alpha$, between wind vectors at the levels of 10 and $300 \mathrm{~m}$, equal to about $30^{\circ}$. The angle $\alpha$ for hodographs during Events 4, 7, 8 and 10, 11 is very small, implying cold-air advection. The angle $\alpha$ during event E3 is larger, equal to about $45^{\circ}$.

Profiles of the Richardson $R i$ are depicted in Fig 3, and typically, $R i$ is small near the surface and increases with height (Sorbjan 2012). According to Sorbjan (2010), thermal stability in the stable boundary layer differs for individual layers, and can be classified based on the local values of the Richardson number. Specifically, the layers can be "near-neutral" when $0<R i<0.02$, "weakly stable" when $0.02<R i<0.12$, "very stable" when $0.12<R i<0.7$, and "extremely stable" when $R i>0.7$. When $R i<0.7$, a layer is considered turbulent. Otherwise, in the "extremely stable" case, there is no turbulence, or it appears sporadically.

As it follows from Fig. 3, during Events 1 and 2, the values of the Richardson number were very small, indicating "weak stability" in the entire boundary layer. During Event 3 (strong surface cooling), the largest value of $R i$ appeared at level $10 \mathrm{~m}$, and decreased with height. Thus, the layer close to the surface was "extremely stable". During Events 4, 8, 9, the lower portion of the boundary layer can be classified as "weakly stable", and the upper part as "very stable". During Event 5, thermal stratification was "weakly stable" or "very stable" near the underlying surface, and "extremely stable" above the level of $200 \mathrm{~m}$. During Events 6 and 7, the entire 300-m layer can be classified as "weakly stable".

The boundary layer during Event 10 reached the "extremely stable" state at the level of 100 $\mathrm{m}$, and during Event 11, the layer below level of $100 \mathrm{~m}$ was "weakly stable" or "very stable". The 100-200 m layer was "extremely stable". During Event 12, the layer below the level 150 was "weakly stable" or "very stable", and the uppermost 200-m layer was "extremely stable". 


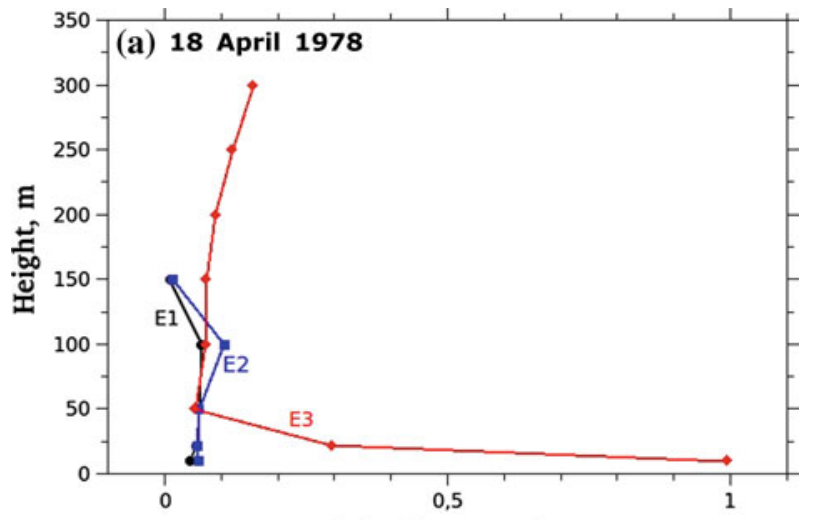

Richardson number
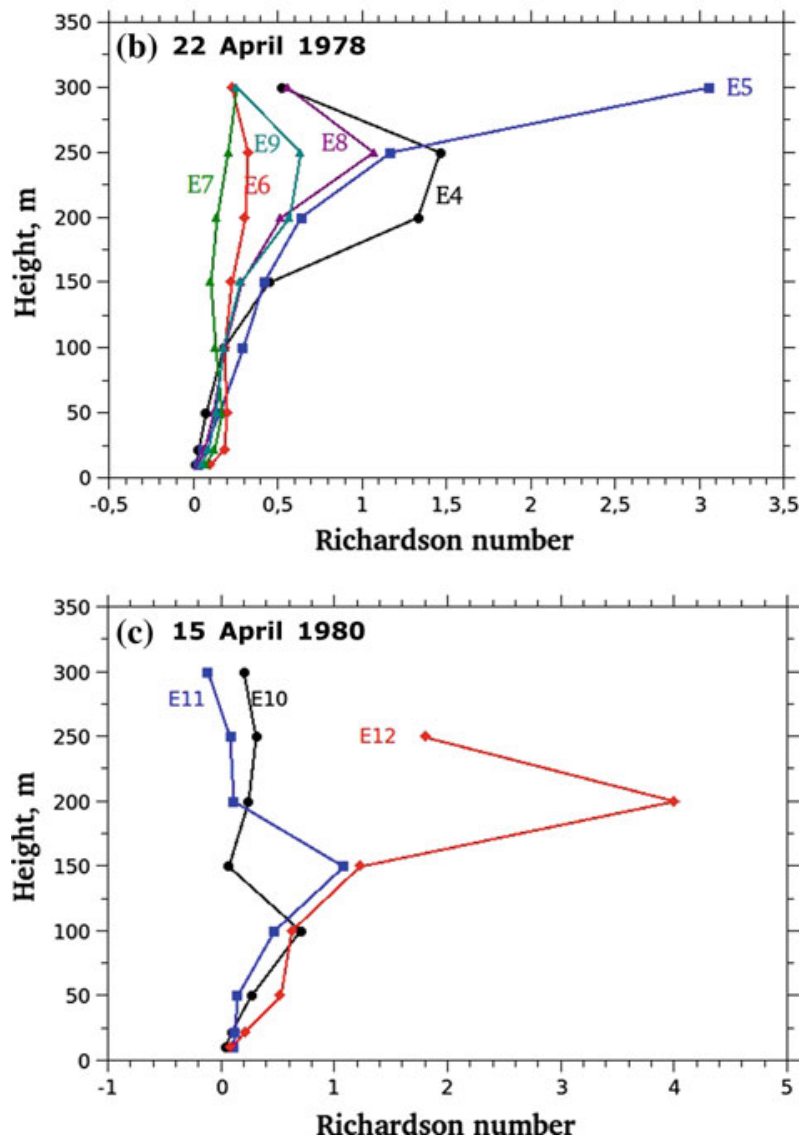

Fig. 3 Profiles of the Richardson number during: a Events E1-E3, b Events E4-E9, c Events E10-E12. The peak of $R i$ appears at levels where the wind shear is smallest 


\subsection{Fluxes and Variances}

The profiles of the second-order moments are depicted in Figs. 4, 5, 6 and 7. Hunt et al. (1985) used 20-min averages, which include the wave scales. For comparison, Sorbjan and
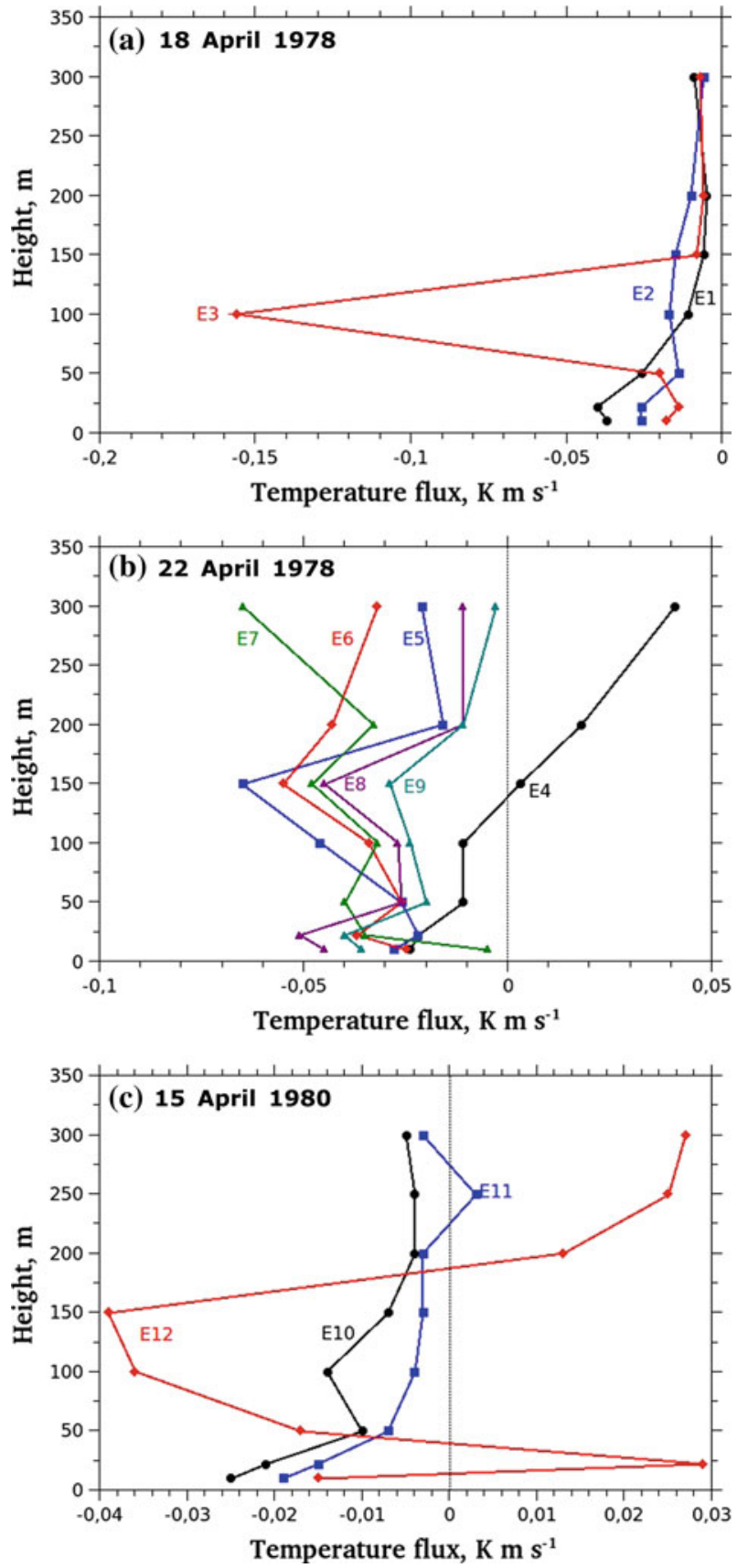

Fig. 4 Profiles of the temperature flux during: a Events E1-E3, b Events 4-9, c Events E10-E12 


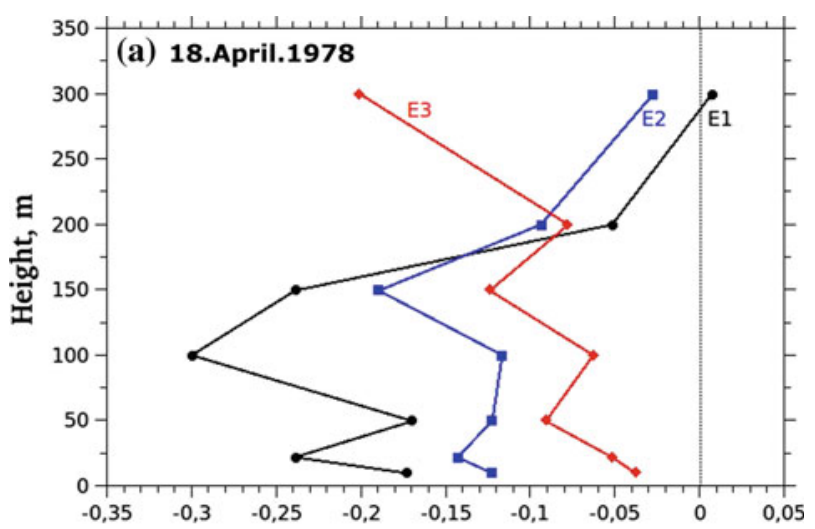

Momentum flux, $\mathrm{m}^{2} \mathrm{~s}^{-2}$
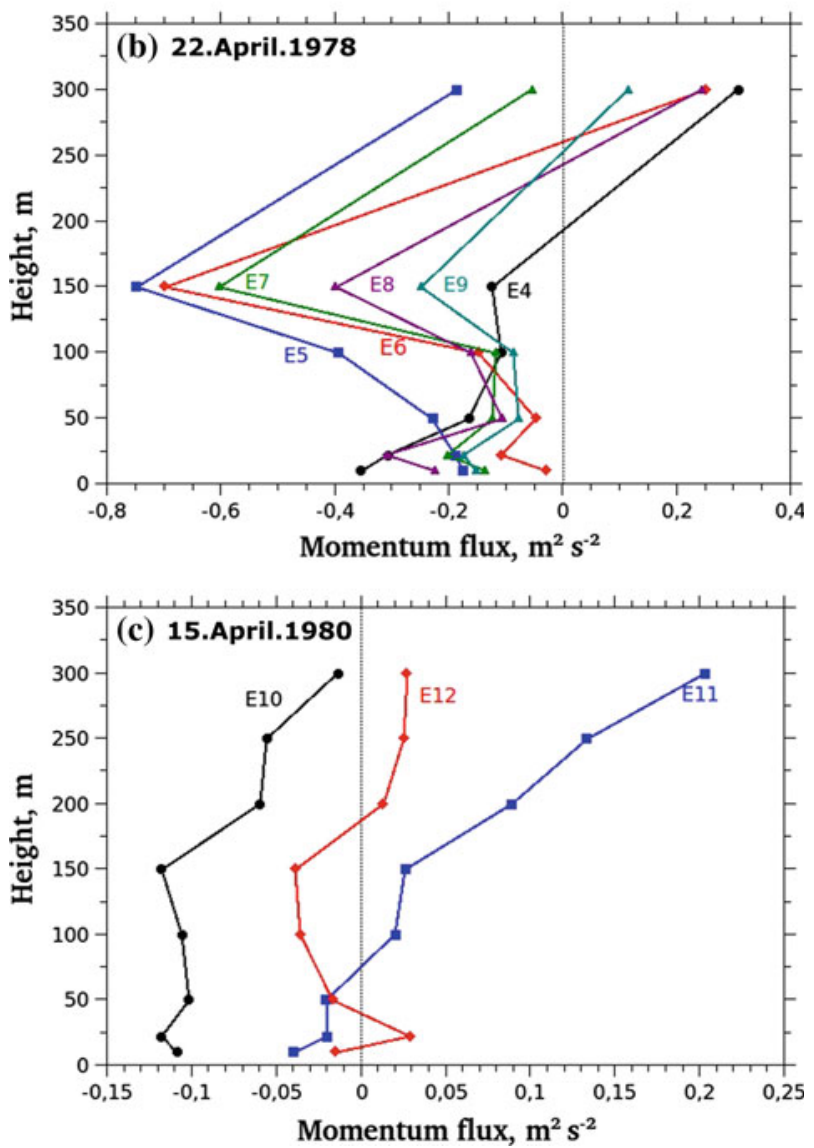

Fig. 5 Profiles of the momentum flux during: a Events E1-E3, b Events E4-E9, c Events E10-E12

Grachev (2010) employed variances and covariances calculated based on 1-h averaging and frequency integration of spectra and cospectra. Figure 4 shows the vertical profiles of the temperature flux $H=\overline{w^{\prime} \theta^{\prime}}$. Note that there were no data available for the level of $250 \mathrm{~m}$ 

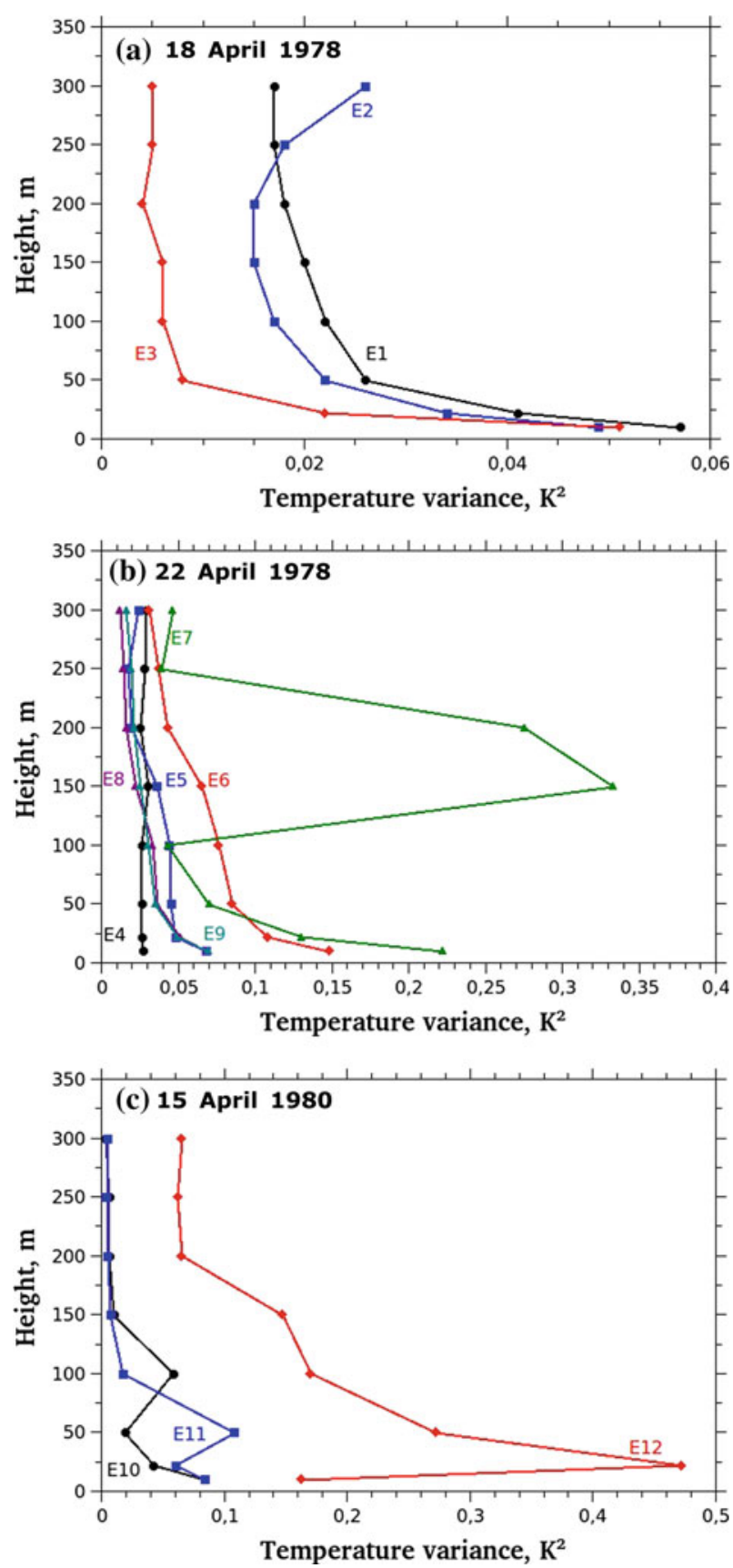

Fig. 6 Profiles of the temperature variance during: a Events E1-E3, b Events E4-E9, c Events E10-E12

during Events 1-9. Profiles during Events 1, 2, 10 and 11 have the expected appearance: values increase with height from the most negative values near the surface to near-zero at the top. There is an outlier (negative) at the level of $100 \mathrm{~m}$ in the E3 profile. During Event 

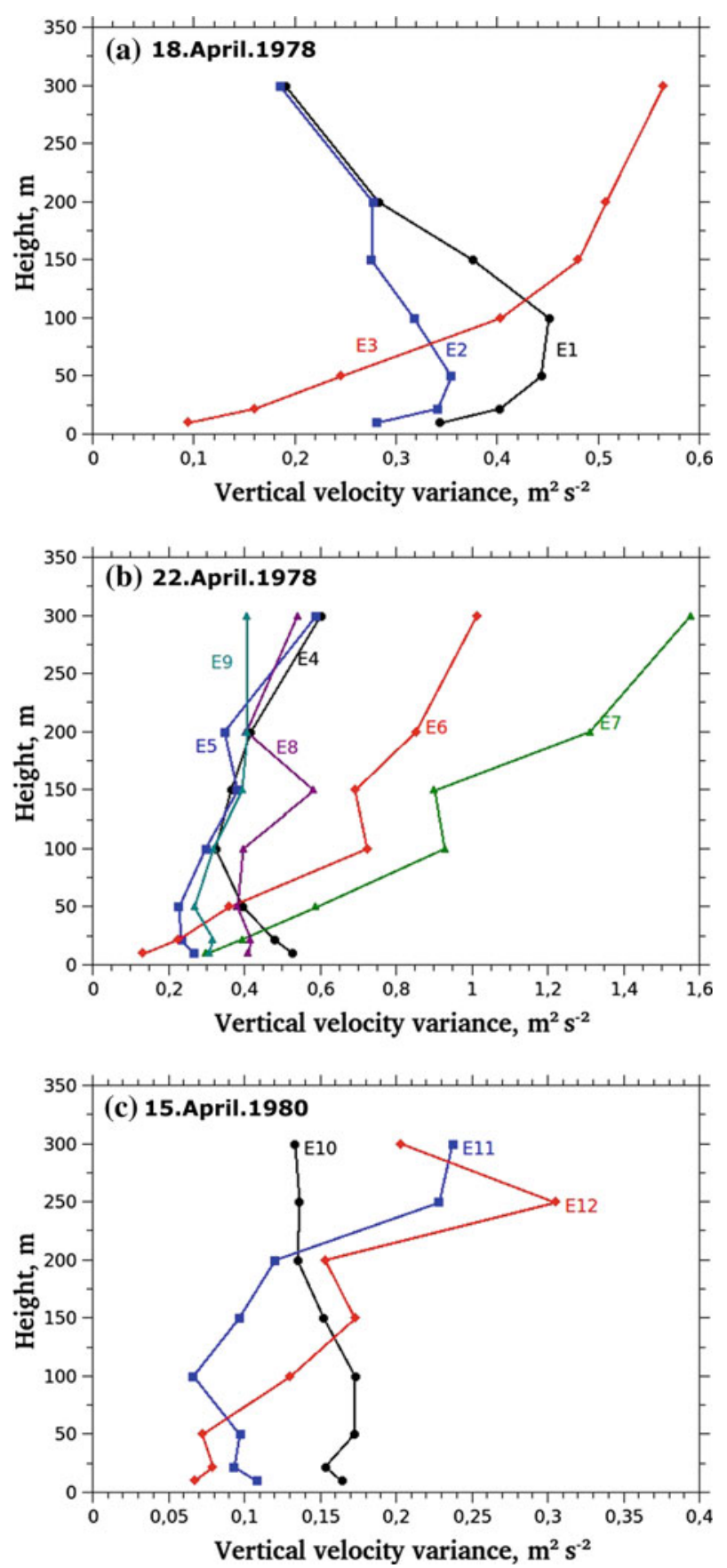

Fig. 7 Profiles of the vertical velocity variance during: a Events E1-E3, b Events E4-E9, c Events E10-E12

4, the temperature flux increased with height, and was positive above $150 \mathrm{~m}$. According to $\mathrm{Lu}$ et al. (1983), the heat flux could be counter-gradient due to the effects of internal waves. 
During Events 5-9, the temperature-flux profiles generally decrease with height within the lowest $150 \mathrm{~m}$ layer, and increase with height in the layer 150-200 m. Above the level of $200 \mathrm{~m}$, heat fluxes are nearly constant during events E5, E8, E9, increase with height during event E6, or decrease during E7, which could be associated with the effects of the gravity waves. The temperature-flux profile during E12 (strong cold-air advection near the surface) is highly irregular with negative and positive outliers. The positive values are counter-gradient, and most likely caused by wave activity.

Profiles of the momentum flux $\tau=\overline{u^{\prime} w^{\prime}}$ are shown in Fig. 5. Note that there were no available data for the level of $250 \mathrm{~m}$ during Events 1-3, and for the levels of 200 and 250 $\mathrm{m}$ during Events 4-9. Profiles during Events 1, 2, 10 have the expected form, where values generally increase with height, from the most negative values near the surface to near-zero values at the top. During Event 3 in Fig. 5a, profile values slightly decreased with height. Profiles in Fig. 5b (Events 4-9) are highly irregular, which can be associated with the effects of the gravity waves. The momentum flux during Event 11 in Fig. $5 \mathrm{c}$ was positive above the 50-m level.

Profiles of the temperature variance are presented in Fig 6, with profiles during Events 1-6 and 8-11 having the expected form. Values decrease with height from positive near the surface to near-zero at the top. During Event 7, effects of internal wave motion are noticeable, as increased values of the variance appear in the layer 100-250 m. During Event 12, increased values of the temperature variances are present above the level of $22 \mathrm{~m}$.

Profiles of the vertical velocity variance are shown in Fig 7. It is expected that the vertical velocity variance decreases with height, from positive values near the surface to near-zero values at the top. During Events 1 and 2, the variance first increases with height up to the level of $50 \mathrm{~m}$, and then decreases with height. The profile values during Events 3-9 increase with height, which can be associated with the effects of gravity waves. During Events 10-12, the variances are nearly constant with height. Note that data at the level of $250 \mathrm{~m}$ were not available during Events 1-9.

\section{Gradient-Based Similarity Formulation}

\subsection{Definition of Scales and Universal Functions}

In this section, we will consider a similarity theory approach for the description of turbulence in stable conditions. Sorbjan (2010) introduced several gradient-based, local scaling systems for the stably stratified boundary layer, and also derived similarity functions using data collected during the SHEBA field program in the Arctic (Grachev et al. 2008). We employ two local scaling systems in this study. The first one, referred to as $\sigma_{\mathrm{w}}$ scaling, is defined by the following set of local scales,

$$
\begin{aligned}
& L_{\mathrm{w}}=\frac{\sigma_{\mathrm{w}}}{N}, \\
& U_{\mathrm{w}}=L_{\mathrm{w}} N \equiv \sigma_{\mathrm{w}}, \\
& T_{\mathrm{w}}=L_{\mathrm{w}} \Gamma \equiv \frac{N \sigma_{\mathrm{w}}}{\beta},
\end{aligned}
$$

where $\sigma_{\mathrm{w}}$ is the standard deviation of the vertical velocity. The length scale $L_{\mathrm{w}}$ in (1a) can be interpreted as the vertical distance by which a parcel of air moves when its kinetic energy is converted to work against the buoyancy force (e.g. Sorbjan 2008). 
The universal similarity functions for the $\sigma_{\mathrm{w}}$ scaling were found to be functions of the Richardson number of following form,

$$
\begin{aligned}
& \frac{\tau}{U_{\mathrm{w}}^{2}}=0.72 \frac{\left(1+450 R i^{2}\right)}{\left(1+300 R i^{2}\right)^{3 / 2}}, \\
& -\frac{H}{U_{\mathrm{w}} T_{\mathrm{w}}}=0.80 R i^{1 / 2} \frac{\left(1+450 R i^{2}\right)}{\left(1+250 R i^{2}\right)^{3 / 2}}, \\
& \frac{\sigma_{\theta}}{T_{\mathrm{w}}}=4.25 R i^{1 / 2} \frac{\left(1+450 R i^{2}\right)^{1 / 2}}{\left(1+250 R i^{2}\right)^{1 / 2}} .
\end{aligned}
$$

The above expressions are valid throughout the entire stable boundary layer, and in the range $R i<0.7$. The value $R i=0.7$ can be considered as an effective critical Richardson number. Note that the dimensionless moments in (2) depend on height $z$, since the Richardson number is a function of height.

The second set of local scales, referred to as $\sigma_{\theta}$ scaling, is defined as follows,

$$
\begin{aligned}
L_{\theta} & =\frac{\beta \sigma_{\theta}}{N^{2}}, \\
U_{\theta} & =L_{\theta} N \equiv \frac{\beta \sigma_{\theta}}{N}, \\
T_{\theta} & =L_{\theta} \Gamma \equiv \sigma_{\theta},
\end{aligned}
$$

where $\sigma_{\theta}$ is the standard deviation of the temperature fluctuations. The similarity functions for the $\sigma_{\theta}$ scaling have the following form (Sorbjan 2010),

$$
\begin{aligned}
\frac{\tau}{U_{\theta}^{2}} & =0.04 \frac{\left(1+2500 R i^{2}\right)}{R i\left(1+300 R i^{2}\right)^{3 / 2}}, \\
-\frac{H}{U_{\theta} T_{\theta}} & =0.044 \frac{\left(1+2500 R i^{2}\right)}{R i^{1 / 2}\left(1+250 R i^{2}\right)^{3 / 2}}, \\
\frac{\sigma_{\mathrm{w}}}{U_{\theta}} & =0.24 \frac{\left(1+2500 R i^{2}\right)^{1 / 2}}{R i^{1 / 2}\left(1+450 R i^{2}\right)^{1 / 2}} .
\end{aligned}
$$

The above expressions also apply throughout the entire stable boundary layer, and are valid in the range $R i<0.7$. Note that $\sigma_{\mathrm{w}} / U_{\theta}=\left(\sigma_{\theta} / U_{\mathrm{w}}\right)^{-1}$.

Sorbjan (2010) and Sorbjan and Grachev (2010),also provided the analytical expressions indicating the dependence of the MO local stability parameter $z / \Lambda_{*}$, the flux Richardson number $R f$, and the correlation coefficient $r_{\mathrm{w} \theta}$ between the vertical velocity and temperature, on the Richardson number $R i$,

$$
\begin{aligned}
\frac{z}{\Lambda_{*}} & =\frac{R i}{0.9} \frac{\left(1+300 R i^{2}\right)^{9 / 4}}{\left(1+250 R i^{2}\right)^{3 / 2}}, \\
R f & =\frac{R i}{0.9} \frac{\left(1+300 R i^{2}\right)^{3 / 2}}{\left(1+250 R i^{2}\right)^{3 / 2}}, \\
r_{\mathrm{w} \theta} & =-0.2\left(1+2500 R i^{2}\right)^{1 / 2} \frac{\left(1+450 R i^{2}\right)^{1 / 2}}{\left(1+250 R i^{2}\right)^{3 / 2}},
\end{aligned}
$$

where $\Lambda_{*}=-\tau^{3 / 2} /(\kappa \beta H)$ is the local Obukhov length, $R f=-\beta H /(\tau S)$, and $r_{\mathrm{w} \theta}=$ $H /\left(\sigma_{\mathrm{w}} \sigma_{\theta}\right)$ is the correlation coefficient between temperature and the vertical velocity.

It should be noted that within the local extension of the MO similarity theory, the local scales for velocity, temperature and length, $U_{*}, \vartheta_{*}$ and $\Lambda_{*}$, are based on local values of 
two moments, the momentum and heat fluxes $\tau(\mathrm{z})$ and $H(z)$ (e.g., Sorbjan 1986a,b). The local MO theory assumes that dimensionless moments are functions of the parameter $z / \Lambda_{*}$. A general form of the similarity function in stable conditions can be derived assuming the $z-$ less regime (e.g., Sorbjan 1986a,b), which implies that the dimensionless moments approach constant values for sufficiently large values of $z / \Lambda_{*}$.

Since the MO similarity functions and the parameter $z / \Lambda_{*}$ include the same parameters ( $\tau$ and $H$ ), the self-correlation problem arises. For comparison, the gradient-based similarity scales (1) and (3) are based on two independent parameters, on a variance $\left(\sigma_{\mathrm{w}}\right.$ or $\left.\sigma_{\theta}\right)$, and on $N$, while the similarity functions depend on the Richardson number $R i=N^{2} / S^{2}$ (which introduces the third independent parameter $S$ ). As the result, the effects of self-correlation are less severe.

\subsection{Dimensionless Characteristics of Turbulence}

Figures 8 and 9 show a comparison of the dimensionless fluxes and variances observed at $\mathrm{BAO}$, with their estimates, based on the gradient-based similarity functions, described by Eqs. 2, 4, and obtained from SHEBA data. Symbols in Fig. 8 represent the observational moments normalized by the $\sigma_{\mathrm{w}}$ scales: $\tau / U_{\mathrm{w}}^{2}, H /\left(U_{\mathrm{w}} T_{\mathrm{w}}\right)$, and $\sigma_{\theta} / T_{\mathrm{w}}$, for weak, moderate and strong waves, and also for the unspecified wave cases. Similar symbols in the Fig. 9 display the moments, normalized by the $\sigma_{\theta}$ scales: $\tau / U_{\theta}^{2}, H /\left(U_{\theta} T_{\theta}\right)$, and $\sigma_{\mathrm{w}} / T_{\theta}$.

Referring to the SHEBA similarity functions (2), (4), and (5), it should be mentioned that there is no clear evidence of the gravity wave presence or absence during the SHEBA experiment. It could only be stated that the SHEBA dataset is not affected by gravity waves typically generated by obstacles, such as hills, tall plant canopies, and slopes, since the experimental site was located a few hundred $\mathrm{km}$ from land, over an ice surface with an almost unlimited and extremely uniform fetch. Generally, cospectra of surface fluxes observed during SHEBA are less contaminated by mesoscale motions as compared with data from other sites. The low-frequency disturbances in spectra and cospectra during very stable conditions were often well-pronounced, except spectra for the $w$ component (Grachev et al. 2005, 2008). A potential source of such behaviour could be Arctic leads (e.g. Mauritsen et al. 2005), and the passage of snowstorms (e.g. Bosart and Sanders 1986).

The empirical points in Fig. 8 follow the similarity curves surprisingly well. Departures from the curves in Fig. 8a, b are larger for larger values of the Richardson number. The largest departures for the fluxes can be observed for $R i>0.2$, for weak waves, strong waves, and in cases with unspecified wave activities. The temperature variance, in Fig. 8c, agrees with the similarity curve very well, except for a few outliers (for strong wave cases and also unspecified cases) that exceed the theoretical values, especially around $R i=0.1$. The dependence between the intensity of the gravity waves and the departures of the empirical points from similarity curves cannot be clearly established in Fig. 8.

The overall agreement of the empirical points with the similarity curves in Fig. 9 is quite good, nonetheless not as good as obtained for the $\sigma_{\mathrm{w}}$ scaling in Fig. 8. The largest departures in Fig. 9a, b can be observed for $R i \sim 0.1$ during strong wave cases and in unspecified cases. The temperature variance agrees with the similarity curve very well (note that Figs. $8 \mathrm{c}$ and 9c are symmetrical). As in Fig. 8, the dependence between the intensity of the gravity waves and the extent of the departure of the empirical points for all moments from the similarity curves cannot be clearly established.

A dependence of the remaining dimensionless characteristics of turbulence, i.e. the stability parameter $z / \Lambda_{*}$, the flux Richardson number $R f$, and the correlation coefficient $r_{\mathrm{w} \theta}$, 

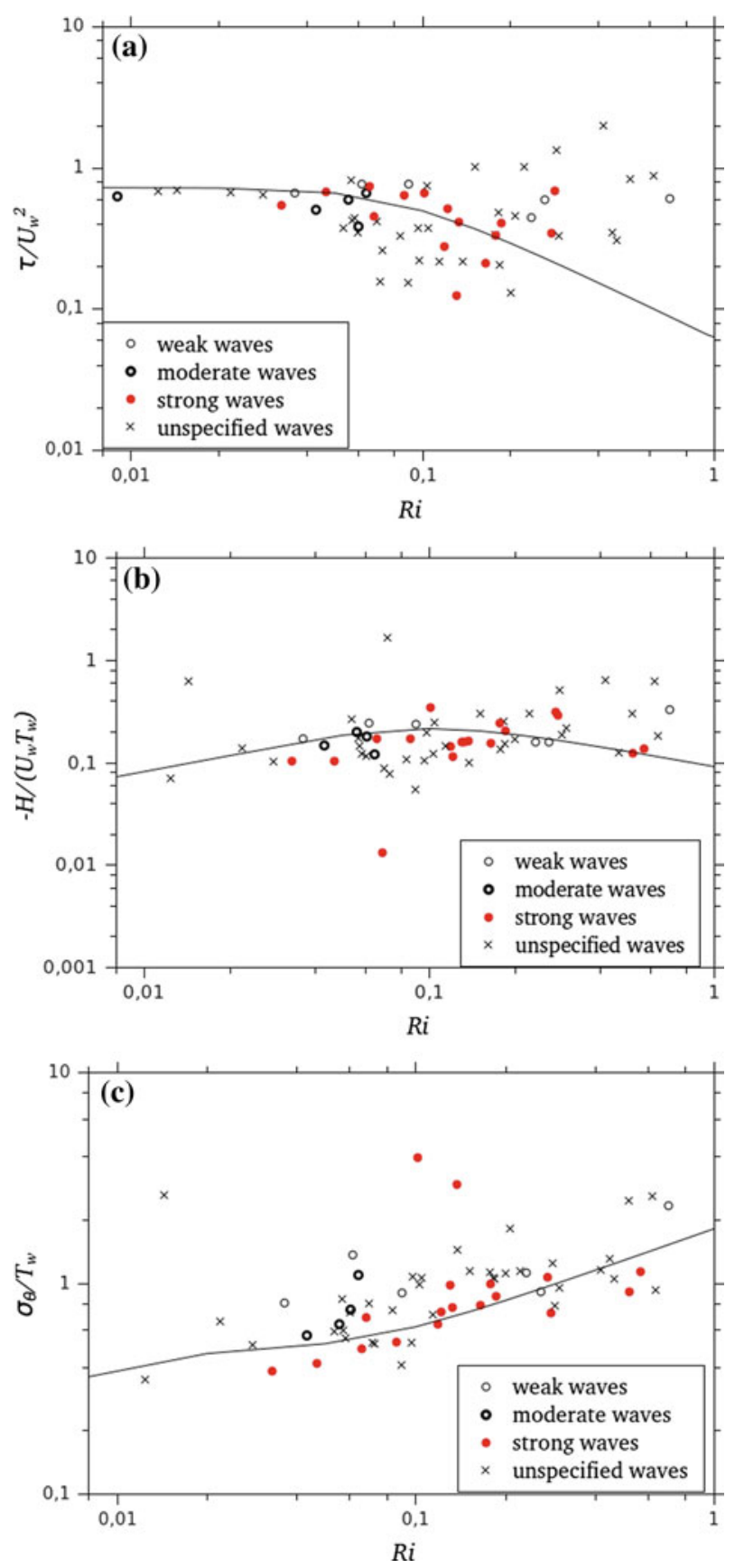

Fig. 8 A dependence of the dimensionless moments (the $\sigma_{\mathrm{W}}$ scaling) on the Richardson number Ri: a the momentum flux $\tau / U_{\mathrm{w}}^{2}$, b the temperature flux $H /\left(U_{\mathrm{w}} T_{\mathrm{w}}\right), \mathbf{c}$ the standard deviation $\sigma_{\theta} / T_{\mathrm{W}}$

on the Richardson number $R i$, is depicted in Fig. 10 a-c. Here, the similarity functions (5a)(5c) are also displayed as continuous curves. The general agreement of the empirical values (points) with the curves is quite good. At $R i>0.2$, the points representing $z / \Lambda_{*}$ are located 

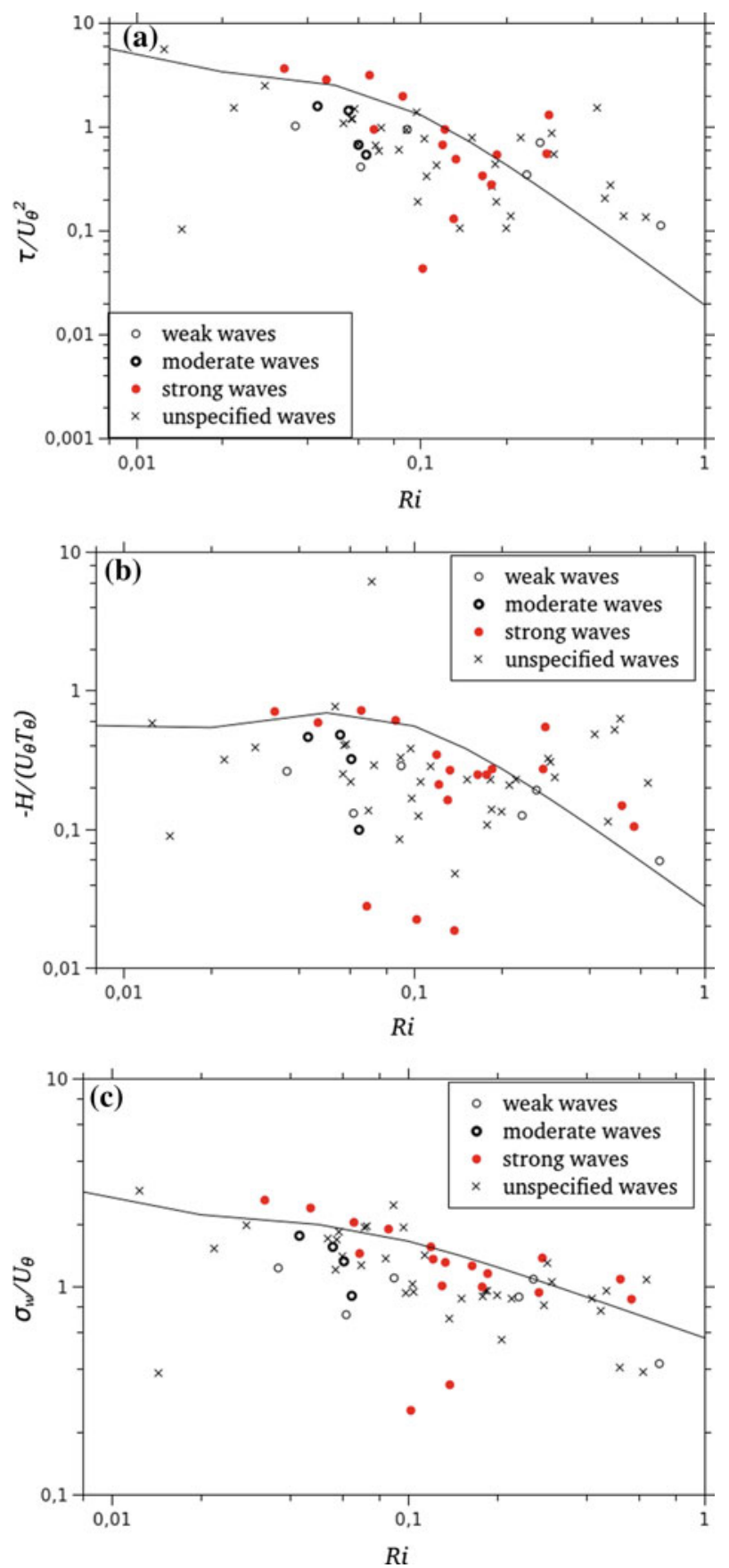

Fig. 9 A dependence of the dimensionless moments (the $\sigma_{\theta}$ scaling) on the Richardson number Ri: a the momentum flux $\tau / U_{\theta}^{2}$, b the temperature flux $H /\left(U_{\theta} T_{\theta}\right)$, $\mathbf{c}$ the standard deviation $\sigma_{\mathrm{W}} / T_{\theta}$ 

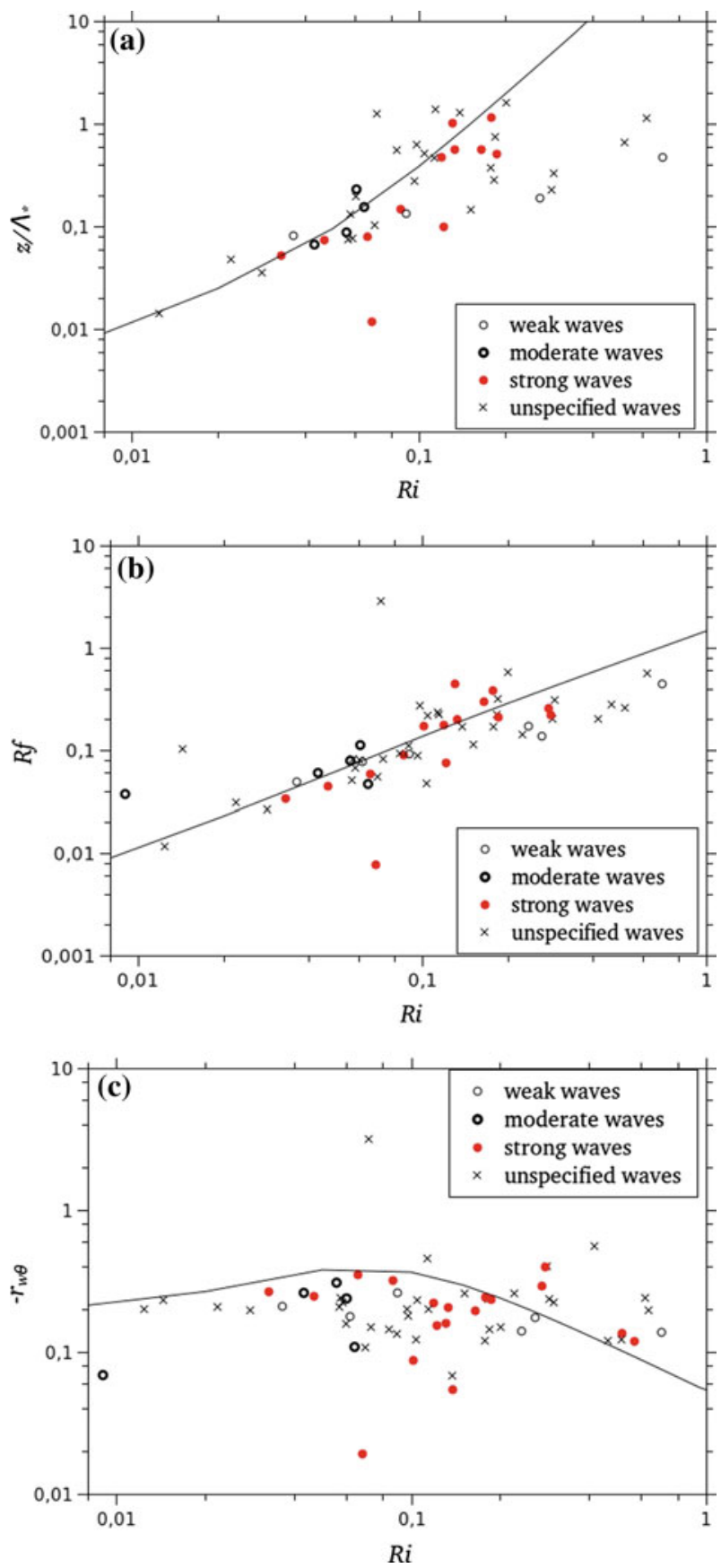

Fig. 10 A dependence of the dimensionless characteristics of turbulence on the Richardson number Ri: a the stability parameter $z / \Lambda_{*}, \mathbf{b}$ the flux Richardson number $R f$, $\mathbf{c}$ the correlation coefficient $r_{\mathrm{w} \theta}$ 
below the similarity curve. There is no clear dependence of the departures from the curves and the intensity of gravity waves, except for a few outliers located around $R i \sim 0.1$.

\section{Conclusions}

In order to investigate the effects of gravity waves on similarity relationships in the stable boundary layer, we re-examined the data set of Hunt et al. (1985), collected on the 300-m tower of the Boulder Atmospheric Observatory (BAO) during three April nights in 1978 and 1980. The dataset included 12 events, with wave motion present on all occasions, and were classified by HKG-85 as cases with "weak waves", moderate waves", and strong waves". The intensity of waves for seven events was not directly specified.

The considered momentum and temperature fluxes, as well as the temperature and vertical velocity variances in the 300 -m layer, were scaled by two gradient-based similarity scaling systems, and compared with similarity functions of the Richardson number, obtained during the SHEBA experiment in the Arctic, with no influence of gravity currents and topographical factors. The first scaling system was based on local length values of the form $L_{\mathrm{w}}=\sigma_{\mathrm{w}} / N$, with the second one based on $L_{\theta}=\beta \sigma_{\theta} / N^{2}$.

Our analysis showed that empirical points agreed favourably with the gradient-based similarity functions for both scaling systems (2), (4) and (5). Significant departures from the theoretical predictions appeared as outliers, but the overall dependency of dimensionless moments on the Richardson number was maintained. The dependence between the intensity of the gravity waves and the extent of departures for all moments could not be clearly established.

Acknowledgments This work has been supported by the US National Science Foundation grant ATM0938293, and by the Polish National Science Centre grant N N307 05 7240. The authors are grateful for a helpful technical assistance of Dr. Marek Kubicki.

Open Access This article is distributed under the terms of the Creative Commons Attribution License which permits any use, distribution, and reproduction in any medium, provided the original author(s) and the source are credited.

\section{References}

Blumen W, Banta R, Burns SP, Fritts DC, Newsom R, Poulos GS, Sun J (2001) Turbulence statistics of a KelvinHelmholtz billow event observed in the night-time boundary layer during the cooperative atmospheresurface exchange field program. Dyn Atmos Oceans 34:189-204

Bosart LF, Sanders F (1986) Mesoscale structure in the megapolitan snowstorm of 11-12 February 1983. Part III: A large amplitude gravity wave. J Atmos Sci 43:924-939

Caughey SJ, Readings CJ (1975) An observation of waves and turbulence in the earth's boundary layer. Boundary-Layer Meteorol 9:279-296

Cuxart J, Yague C, Morales G, Terradellas E, Orbe J, Calvo J, Fernandz A, Soler MR, Infante C, Buenestado P, Espinalt A, Joergensen HE, Rees JM, Vila J, Redondo JM, Cantalapiedra IR, Conangla L (2000) Stable atmospheric boundary layer in Spain (SABLES 98): a report. Boundary-Layer Meteorol 96:337-370

Einaudi F, Finnigan JJ (1993) Wave-turbulence dynamics in the stably stratified boundary layer. J Atmos Sci 50:1841-1864

Grachev AA, Fairall CW, Persson POG, Andreas EL, Guest PS (2005) Stable boundary layer regimes: the SHEBA data. Boundary-Layer Meteor 116:201-235

Grachev AA, Andreas EL, Fairall W, Guest PS, Persson POG (2008) Turbulent measurements in the stable atmospheric boundary layer during SHEBA: ten years after. Acta Geophys 56(1):142-166

Hunt JCR, Kaimal JC, Gaynor JE (1985) Some observations of turbulence structure in stable layers. Q J R Meteorol Soc 111:793-815 
Lu N-P, Neff WD, Kaimal JC (1983) Wave and turbulence structure in a disturbed nocturnal inversion. Boundary-Layer Meteorol 26:141-155

Mahrt L, Richardson S, Seaman N, Stauffer D (2012) Turbulence in the nocturnal boundary layer with light and variable winds. Q J R Meteorol Soc 138(667):1401-1680

Mauritsen T, Svensson G, Grisogono B (2005) Wave flow simulations over Arctic leads. Boundary-Layer Meteorol 117:259-273

Nappo C (1991) Sporadic breakdowns of stability in the PBL over simple and complex terrain. Boundary-Layer Meteorol 54:69-87

Rees JM, Denholm-Price JC, King JC, Anderson PS (2000) A climatological study of internal gravity waves in the atmospheric boundary layer overlying the Brunt Ice Shelf, Antarctica. J Atmos Sci 57:511-526

Smedman AS (1988) Observations of multi-level turbulence structure in a very stable atmospheric boundary layer. Boundary-Layer Meteorol 44:231-253

Sorbjan Z (1986a) On similarity in the atmospheric boundary layer. Boundary-Layer Meteorol 34:377-397

Sorbjan Z (1986b) Local similarity of spectral and cospectral characteristics in the stable-continuous boundary layer. Boundary-Layer Meteorol 35:257-375

Sorbjan Z (2008) Local scales of turbulence in the stable boundary layer. Boundary-Layer Meteorol 127(2):261-271

Sorbjan Z (2010) Gradient-based scales and similarity laws in the stable boundary layer. Q J R Meteorol Soc 136:1243-1254

Sorbjan Z (2012) A study of the stable boundary layer based on a single-column model. Boundary-Layer Meteorol 142:33-53

Sorbjan Z, Grachev AA (2010) An evaluation of the flux-gradient relationship in the stable boundary layer. Boundary-Layer Meteorol 135(3):385-405

Sun J, Burns SP, Lenschow DH, Banta R, Newsom R, Coulter R, Frasier S, Ince T, Nappo C, Cuxart J, Blumen W, Lee X, Hu X-Z (2002) Intermittent turbulence associated with density current passage in the stable boundary layer. Boundary-Layer Meteorol 105:199-219

Turner JS (1983) Buoyancy effects in fluids. Cambridge University Press, Cambridge, UK, 412 pp

Yagüe C, Maqueda G, Rees JM (2001) Characteristics of turbulence in the lower atmosphere at Halley IV station, Antarctica. Dyn Atmos Oceans 34:205-223 